\title{
Global Weather States and Their Properties from Passive and Active Satellite Cloud Retrievals
}

\author{
GEORGE TSELIOUDIS
}

NASA GISS, and Department of Applied Physics and Applied Mathematics, Columbia University, New York, New York, and Research Centre for Atmospheric Physics and Climatology, Academy of Athens, Athens, Greece

\author{
WILLIAM RosSOW
}

NOAA/CREST, City College of the City University of New York, New York, New York

YUANCHONG ZHANG

NASA GISS, and Department of Applied Physics and Applied Mathematics, Columbia University, New York, New York

\section{DIMITRA KONSTA}

Research Centre for Atmospheric Physics and Climatology, Academy of Athens, Athens, Greece

(Manuscript received 10 January 2013, in final form 11 April 2013)

\begin{abstract}
In this study, the authors apply a clustering algorithm to International Satellite Cloud Climatology Project (ISCCP) cloud optical thickness-cloud top pressure histograms in order to derive weather states (WSs) for the global domain. The cloud property distribution within each WS is examined and the geographical variability of each WS is mapped. Once the global WSs are derived, a combination of CloudSat and Cloud-Aerosol Lidar and Infrared Pathfinder Satellite Observations (CALIPSO) vertical cloud structure retrievals is used to derive the vertical distribution of the cloud field within each WS. Finally, the dynamic environment and the radiative signature of the WSs are derived and their variability is examined. The cluster analysis produces a comprehensive description of global atmospheric conditions through the derivation of $11 \mathrm{WSs}$, each representing a distinct cloud structure characterized by the horizontal distribution of cloud optical depth and cloud top pressure. Matching those distinct WSs with cloud vertical profiles derived from CloudSat and CALIPSO retrievals shows that the ISCCP WSs exhibit unique distributions of vertical layering that correspond well to the horizontal structure of cloud properties. Matching the derived WSs with vertical velocity measurements shows a normal progression in dynamic regime when moving from the most convective to the least convective WS. Time trend analysis of the WSs shows a sharp increase of the fair-weather WS in the 1990s and a flattening of that increase in the 2000s. The fact that the fair-weather WS is the one with the lowest cloud radiative cooling capability implies that this behavior has contributed excess radiative warming to the global radiative budget during the 1990s.
\end{abstract}

\section{Introduction}

To better understand the process by which the atmospheric circulation produces clouds, it is important to map the major cloud regimes and their variability and understand how they relate to the corresponding meteorological conditions. The availability of many detailed

Corresponding author address: George Tselioudis, NASA/GISS, Columbia University, 2880 Broadway, New York, NY 10025.

E-mail: gt9@columbia.edu cloud and atmospheric property measurements that cover the entire globe for many years makes it possible to conduct such an evaluation in a statistically robust fashion, rather than for just a few case studies. One approach to such an analysis defines atmospheric weather states (WSs) through the application of cluster analysis techniques to the International Satellite Cloud Climatology Project (ISCCP) cloud top pressure-optical thickness (PC-TAU) joint histograms (Jakob and Tselioudis 2003; Rossow et al. 2005b). The cloud-based WSs have subsequently been used to study cloud-dynamical associations

DOI: 10.1175/JCLI-D-13-00024.1 
of radiation and precipitation variability (Jakob and Tselioudis 2003; Jakob et al. 2005; Rossow et al. 2005b; Tromeur and Rossow 2010; Mekonnen and Rossow 2011; Haynes et al. 2011; Oreopoulos and Rossow 2011; Tselioudis and Rossow 2011; Tselioudis et al. 2010; Lee et al. 2013; Rossow et al. 2013) and to evaluate cloud and radiation variability in climate model simulations (e.g., Williams and Tselioudis 2007; Williams and Webb 2009). The cloud-based WSs were first derived separately for the major climate zones, namely, the tropical and midlatitude zones, because it was easier both to constrain the number of clusters needed to describe the cloud field and to interpret the resulting cloud structures with respect to the known features of the dynamic regime that creates a particular WS. However, it was obvious from the maps of WS distributions (e.g., Oreopoulos and Rossow 2011) that the derived weather states were not confined to the arbitrarily defined boundaries of these climate zones. Furthermore, such boundaries made it harder to examine variability of the weather states with respect to the physical processes that are producing them, as such processes can vary spatially and cross the boundaries of the climate zones. All this pointed to the need to derive weather states for the global domain.

One major question regarding the WSs that were derived using the ISCCP PC-TAU histograms has been the representativeness and the uniqueness of the WS cloud definitions, especially as the uniqueness relates to the vertical distribution of the clouds in each WS. The recent availability of several years of cloud vertical structure (CVS) retrievals from CloudSat and CloudAerosol Lidar and Infrared Pathfinder Satellite Observations (CALIPSO) now makes it possible to investigate the relationship of CVS and the WSs. The study of Zhang et al. (2007) derived tropical cloud clusters applying the same clustering technique to CloudSat histograms of CVSs and found good correspondence and clear physical connections between those clusters and the ones derived from ISCCP PC-TAU histograms in Rossow et al. (2005b). Also, the study of Haynes et al. (2011) identified unique CVSs in the CloudSat retrievals associated with the ISCCP WSs over the Southern Ocean.

In this study, we apply the same clustering methodology that was used in the tropical studies of Jakob and Tselioudis (2003) and Rossow et al. (2005b) in order to derive WSs for the global domain. The cloud property distribution within each weather state is examined and their geographical variability mapped. Various tests are performed to determine the optimum number of WSs and to ensure the statistical robustness of the final set (section 2a). Once the global weather states are derived, the combined CloudSat radar-CALIPSO lidar measurements are classified according to the arrangement of cloud layers in each profile (section 2b). The frequency of each CVS category associated with each matched occurrence of the ISCCP WSs is then compiled. Finally, the large-scale vertical motions from the European Centre for Medium-Range Weather Forecasts (ECMWF) Interim Re-Analysis (ERA-Interim) (Dee et al. 2011) and NCEP/ Department of Energy Global Reanalysis 2 (NRA2) (Kanamitsu et al. 2002), along with the radiative fluxes from the ISCCP flux data (FD) product (Zhang et al. 2004), are compiled to characterize the atmospheric dynamics and cloud-radiative effects of these WSs. Our objective is to not only test the distinctiveness of the WSs, but also to use both passive and active satellite instruments to extend the properties of these states to the associated CVS and large-scale circulation. In addition, we perform a preliminary examination of the variability of the WSs over the recent 26-yr period.

\section{Methodology \\ a. Derivation of global weather states}

In our previous work (Jakob and Tselioudis 2003; Rossow et al. 2005b), the $K$-means clustering algorithm (e.g., Anderberg 1973) was applied to the cloud fraction vector formed from the histograms of PC-TAU (in $7 \times$ 6 intervals, giving a vector dimension of 42) for each 3-hourly, 280-km ISCCP stage D1 grid cell over the period July 1983-June 2009 in four climate zones (tropical, extended tropics, and north and south midlatitudes) to derive optimized WSs (the results can be obtained at http://isccp.giss.nasa.gov/climanal5.html or at http://crest. ccny.cuny.edu/rscg/products.html). Since TAU is only available during daytime in the ISCCP D1 dataset, the derived weather states are also only available for daytime. [See Tan et al. (2013) and Tan and Jakob (2013) for a revised IR-based method that overcomes this limitation for the tropics.] In the cluster analysis, the "best" (optimum) cluster number $K$ is determined objectively by a set of diagnostic checks as described below. The present study extends the previous climate-zone works by applying the analysis to the histograms for the entire global domain.

As $K$ was already 9 for the Northern Hemisphere zone $\left(30^{\circ}-65^{\circ} \mathrm{N}\right.$; cf. Oreopoulos and Rossow 2011), we start the clustering at $K=9$ and run various tests as $K$ is increased. The first test is to check that the clustering procedure "converges," as defined by the maximum value of the square of centroid difference sum between iterations becoming $<0.001$. This quantity is a measure of the changes between iterations in the locations of the cluster centroids in the 42-dimension vector space. The second test is to check that this convergence is insensitive 
to choosing another random set of centroids to initiate the analysis [criterion 1 in Rossow et al. (2005b)], as judged by testing the similarity of the resulting patterns (correlations of the centroid 2D patterns are required to be $>0.8$ ). Sensitivity to the initial set usually indicates that $K$ is too small (this sensitivity test was done three times for the final $K$ ). As the cluster number increases, the centroid 2D patterns at a particular $K$ are cross correlated (WS $\mathrm{W}_{i}$ and $\mathrm{WS}_{j}$, where $i \neq j$ ). If any pair of WSs for a given $K$ has a correlation $>0.8$ [instead of 0.6 in Rossow et al. (2005a) or 0.9 in Williams and Tselioudis (2007)], this indicates the splitting of a cluster from the $K-1$ results, suggesting that $K$ is too large [criterion 2 in Rossow et al. (2005b)]. The third test is to measure the dispersion of all the vectors in each cluster (rms of each vector's distance from the centroid) and look for the $K$ value for which this is a minimum. The last test, which is the complement of the test for cluster splitting, is to cross correlate all of the centroid PC-TAU distribution patterns for $K$ and $K+1$, looking for an indication that a "new" pattern has appeared going from $K$ to $K+1$. If some of the WS patterns for $K$ exhibit correlations $\leq 0.5$ with the WS patterns for $K+1$, this indicates that $K$ is not large enough; if all of the WS patterns for $K$ have correlations $>0.5$ with the patterns for $K+1$, this indicates that $K+1$ is too large.

Based on all these tests, $K=11$ was found to be the best representation of the 26 years of global ISCCP PCTAU histograms. The centroid pattern converged and was not sensitive to the random set of centroids used to initialize the analysis. The results for $K=11$ had the minimum values for both the minimum and the maximum cluster dispersions compared to results for $K=9$, 10 , and 12 . In the cross-correlation tests smaller $K$ results could not match all the patterns for $K=11$, indicating the appearance of new patterns, but the $K=11$ patterns correlated well with all the patterns for $K=12$. For the $K=12$ result, two of the WS patterns were highly correlated with each other (and with one of the patterns for $K=11$ ), indicating a split cluster. All these results justify an optimum $K=11$.

Most of these 11 global weather states (GWSs) strongly resemble the WSs found in the previous analyses in limited latitude zones. For instance, the four GWSs dominated by low clouds are nearly the same as four WSs found for the extended tropics as well as corresponding to three WSs for midlatitudes. The GWSs corresponding to convective anvil clouds in the tropical and extended tropics that also resembled a WS found in the midlatitude zones have been redistributed between two GWSs, one that occurs most frequently at low latitudes and one that occurs most frequently on the poleward edge of the midlatitude zones. The two new GWSs are a distinctly polar cloud property distribution and a WS that results from the split of WSs dominated by cirrus, cumulus, and clear sky. The final test of the distinctiveness of the GWSs is their geographic distribution: as we discuss in more detail in the next section, GWSs that appear to have similar PC-TAU distribution patterns actually occur in different parts of the globe and, as we also show in the next section, are actually different in detail.

We arrange the 11 GWSs in an order that begins with the most frequent deepest convection (smallest PC and largest TAU), through WSs with a large amount of highlevel clouds with moderate TAU and less-deep convection, to states dominated by shallow cumulus, cirrus, and clear sky, to states increasingly dominated by low clouds with less cirrus and clear sky. We also include a WS 12 that accounts for mesoscale regions that are completely clear (see Fig. 2 below for detail). In the evaluation of the uniqueness of these WSs, it is important to note that this ordering of the GWSs was performed entirely by examining the PC-TAU patterns and the geographic distribution of the GWSs (see Fig. 3): after seeing the CVS results we only switched the order of two states in the middle of the sequence, but made no changes after seeing the vertical velocity composite results (see section 3 ).

\section{b. Derivation of CVS categories}

In earlier work (Rossow et al. 2005a; Rossow and Zhang 2010), a statistical model of CVS was developed based on radiosonde humidity profiles, in which each cloud is classified as high $(\mathrm{H})$, middle $(\mathrm{M})$, or low (L) depending on cloud top pressure using the ISCCP categories (where $680 \mathrm{mb}$ separates low-level and midlevel clouds and $440 \mathrm{mb}$ separates midlevel and high-level clouds), where separate cloud layers occurring in the same height category are combined into a single cloud layer defined by the uppermost top and lowermost base [see Wang et al. (2000) for justification of this approach]. Then each profile of cloud layers is classified as singlelayer clouds (called $1 \mathrm{H}, 1 \mathrm{M}$, and 1L), double-layer clouds (HL, HM, and ML), and triple-layer clouds (HML), with an additional $\mathrm{HxMxL}$ for a cloud that is continuous (denoted by " $\mathrm{x}$ ") from high-cloud range to low-cloud range. With the combined CloudSat-CALIPSO dataset ( $C-C$; Mace et al. 2009) we can extend this classification by taking account of physical layer thickness, that is, categorizing each profile by the location of cloud top and base. This adds two new categories to the CVS, namely, $\mathrm{HxM}$ and $\mathrm{MxL}$, split out from the original $\mathrm{H}$ and $\mathrm{M}$ clouds and representing thick cloud layers extending over two of the three pressure layers. Thus, we use a CVS classification with 10 types, 11 including clear sky. Figure 1 is a schematic illustrating these CVS types and showing how we will display the frequency of occurrence 


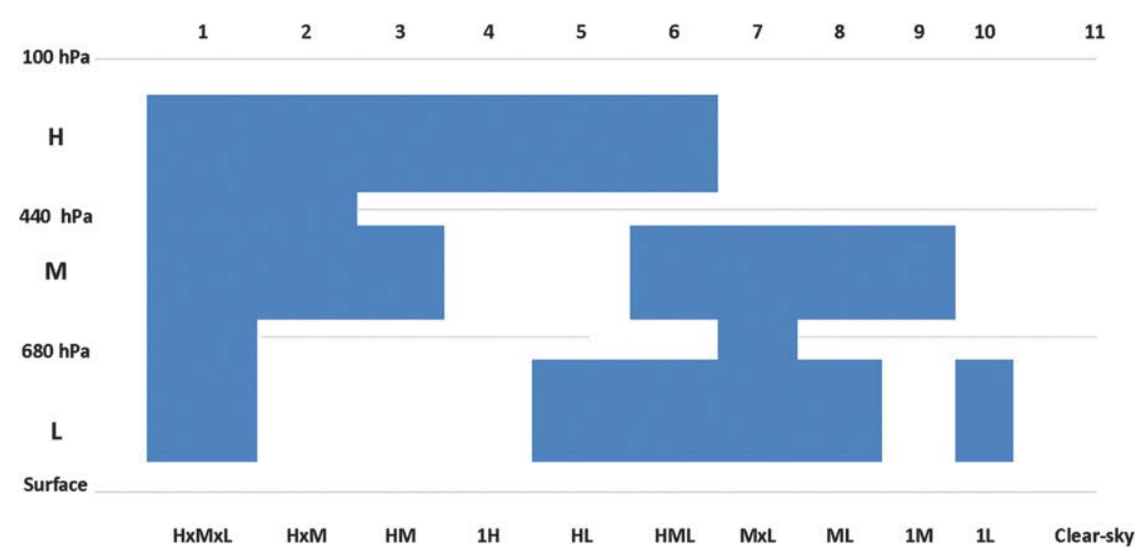

FIG. 1. Schematic of the 11 CVS types (including clear sky as CVS 11). The $x$ axis indicates the layer composition.

of mixtures of different cloud layer profiles obtained from $C-C$ and matched with the GWSs.

For this study, we matched each cloud profile from the most recently reprocessed version of the $C-C$ merged radar-lidar product, the 2B-GEOPROF-lidar of P2_R04 dataset (http://www.cloudsat.cira.colostate.edu/), for the maximum overlap period, July 2006-December 2009. Each $C-C$ cloud profile is classified as one of the 11 types described above (including clear sky, Fig. 1) and then matched with each GWS on 280-km equal-area ISCCP map grid, producing relative frequencies of occurrence (RFO) of each CVS for each GWS. In displaying the results, we show schematically, as in Fig. 1, the RFOs only for those CVS types that occur more than $5 \%$ of the time.

\section{c. Compositing}

We also composite vertical velocities from two reanalyses, ERA-Interim (Dee et al. 2011) and NRA2 (Kanamitsu et al. 2002), matched at 6-h intervals to the GWSs; these values are averaged into the ISCCP equalarea map grid. To establish the radiative signatures of the GWSs derived in this study, the ISCCP FD dataset (Zhang et al. 2004) is matched to the WSs and the cloud radiative effect (CRE) on top-of-atmosphere fluxes is determined for each WS. Since the ISCCP FD dataset is 3-hourly and on the same map grid as the GWSs, the matchup is straightforward.

\section{Results}

\section{a. ISCCP-based global weather states}

The PC-TAU histograms (centroid patterns) for the 11 GWSs are presented in Fig. 2, where the colors indicate the cloud fraction with each combination of PCTAU values, and the relative frequency of occurrence
(RFO) of each WS is shown at the top of each histogram. As described in section 2, the GWS histograms are arranged from the most convective, dominated by high and relatively thick clouds (WSs 1-3), followed by those dominated by middle and relatively thick cloud (WSs 45 ), then by those dominated by high and thin clouds (WS 6 and in part of WS 7), and finally by those dominated by low clouds (WSs 7-11) arranged from optically thinner to thicker low-cloud types. WS 12 is clear sky, namely, those instances when the $2.5^{\circ}$-equivalent equal-area map grid cell is completely cloud-free (only about $2 \%$ of the cases). Figure 3 presents global maps of the average RFO of the GWSs in the same order as in Fig. 2. Finally, Table 1 shows the average PC and TAU and total cloud fraction (CF) determined directly from each of the centroid histograms in Fig. 2.

Examining the GWS PC-TAU histograms (Fig. 2) and the geographic distributions (Fig. 3), we can interpret them as follows. WS 1 represents tropical deep convection, as it includes mostly high ( $\mathrm{PC}<310$ ), optically thick clouds (TAU > 23) and is found primarily along the tropical ITCZ, in the Pacific warm pool and Indian Ocean regions, with smaller populations in the South Pacific and South Atlantic convergence zones and the Amazon and equatorial Africa regions. The less frequent occurrences of WS 1 in the Northern Hemisphere midlatitude storm tracks, concentrated nearer the cyclogenesis regions and along the equatorward edge of this regime, are indicative of the cold frontal convection in the stronger NH cyclones, but this type is also seen occasionally in the weaker SH storms (cf. Haynes et al. 2011). The large amount of deep convective clouds in WS 1 (fraction exceeding 15\% in Fig. 2) is consistent with this WS being associated with the mesoscaleorganized tropical convective systems (cf. Jakob et al. 2005; Rossow et al. 2005b; Tromeur and Rossow 2010; Mekonnen and Rossow 2011). WS 2 includes also 
WS $1 \quad \mathrm{RFO}=4.3 \%$

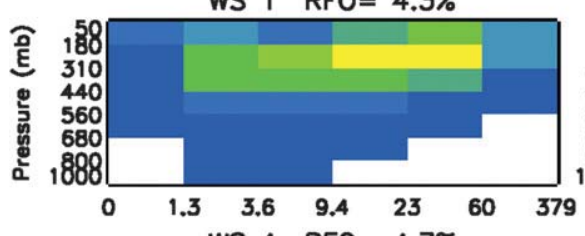

WS 4 RFO $=4.7 \%$
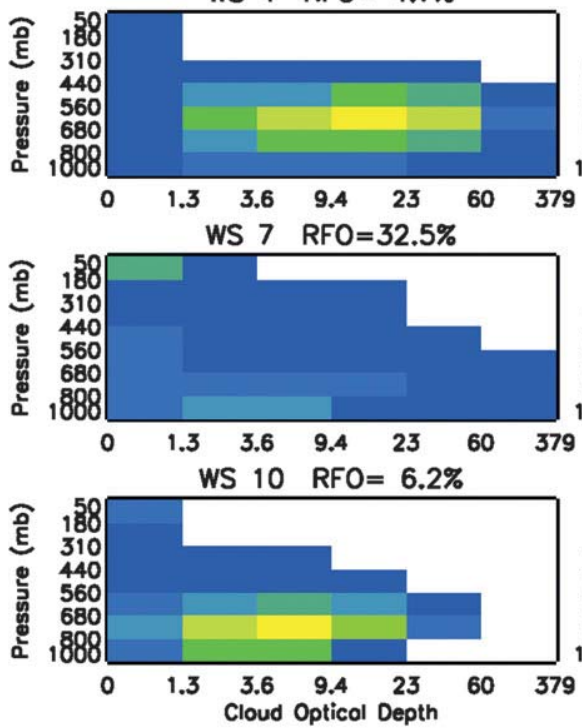

WS $2 \quad \mathrm{RFO}=5.7 \%$

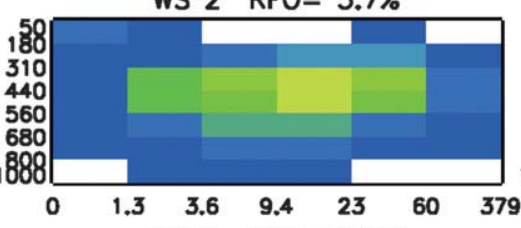

WS 5 RFO $=11.5 \%$
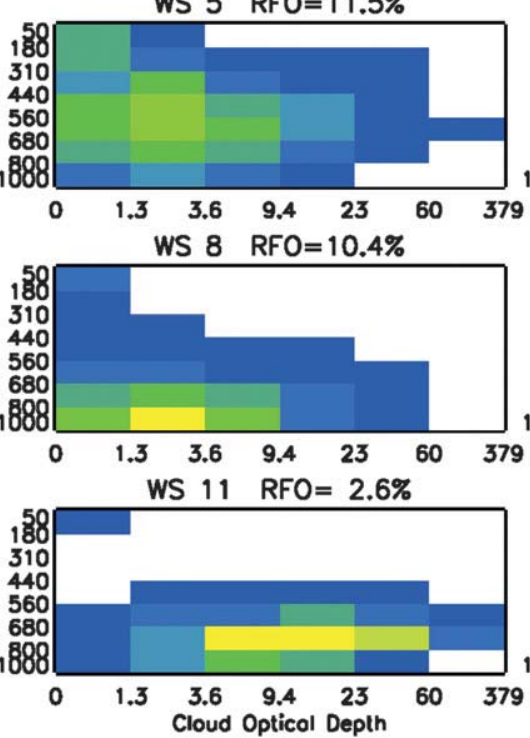

WS $3 \quad \mathrm{RFO}=8.2 \%$

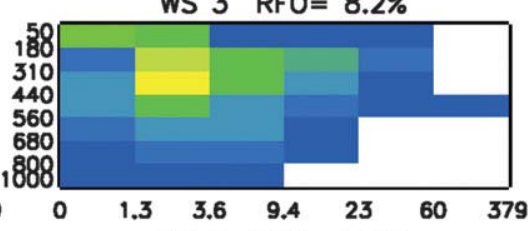

WS $6 \quad \mathrm{RFO}=7.6 \%$
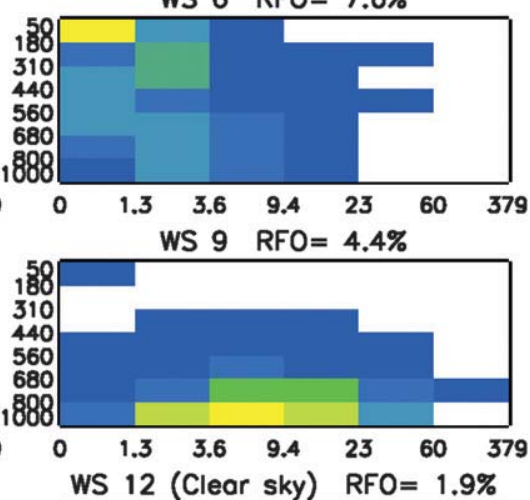

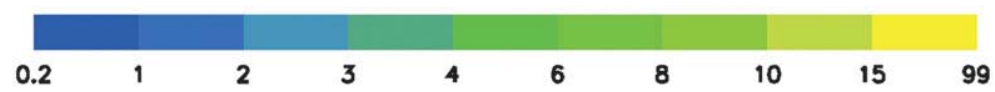

FIG. 2. PC-TAU histograms for the $11 \mathrm{WSs}$ as well as the totally clear sky cluster (WS 12). The WS (cluster) number is indicated at the top of the graph along with the RFO of each WS.

a majority of higher and optically thicker clouds, but these clouds have somewhat lower top heights and are generally thinner than those found in WS 1 (Fig. 2). The geographic distribution of WS 2 shows that these clouds are almost exclusively associated with the midlatitude storm tracks in the two hemispheres, with secondary peaks over Greenland (associated with wintertime cyclones) and the Southeast Asia region (at the poleward edge of the seasonal monsoon). The maps for WS 1 and WS 2 show that the clustering technique has clearly separated the tropical and midlatitude high, thick cloud structures, revealing the lower heights of the midlatitude clouds and suggesting a separation of the more tropicalconvection-like structures in the midlatitude storm tracks (WS 1) from the nimbostratus type of storm clouds (WS 2). WS 3 is dominated by high clouds of low-to-medium optical thickness (Fig. 2) that have the higher cloud tops and the same geographical distribution as WS 1 (with the exception of a secondary peak over the Himalayan plateau). The presence of medium-thickness high clouds and the close geographic association of WS 3 with tropical convection indicate that it represents the stratiform anvil clouds that are part of the mesoscale convective systems, mixed with some isolated convective clouds resulting from less organized and less vigorous convective activity. The fact that the deep convection is small scale is indicated by its low CF and generally lower TAU in the histogram (cf. Rossow et al. 2005a; Tromeur and Rossow 2010; Mekonnen and Rossow 2011). WS 3 also contains some tropical congestus (midlevel cloud tops with large TAU). These three high-cloud WSs are all relatively rare: $\mathrm{WS} 1 \mathrm{RFO}=4.3 \%$, WS $2 \mathrm{RFO}=5.7 \%$, and WS 3 RFO $=8.2 \%$. The mean CF of all three WSs is $>90 \%$, with tropical convection (WS 1) reaching $99 \%$, midlatitude storm clouds (WS 2) reaching 97\%, and anvil cirrus (WS 3) at around 94\% (Table 1). This interpretation of WS 1 and WS 3 is supported by the previous studies focused on tropical WSs (e.g., Rossow et al. 2005a; Jakob et al. 2005).

In the middle-cloud WS categories, WS 4 is dominated by midlevel and moderately optically thick clouds (Fig. 2, Table 1) that occur primarily in the regions poleward of $60^{\circ}$ in both hemispheres (Fig. 3). It is notable that this WS appears to be confined to polar ocean areas. Small amounts are also found on the poleward edges of the midlatitude storm tracks. The minor peak occurrence in 
wS 1

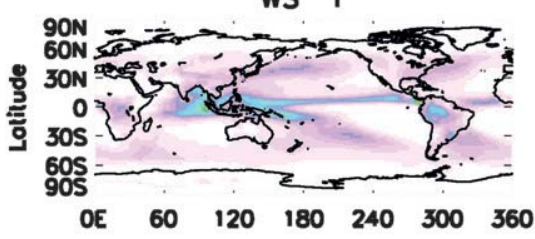

WS 4

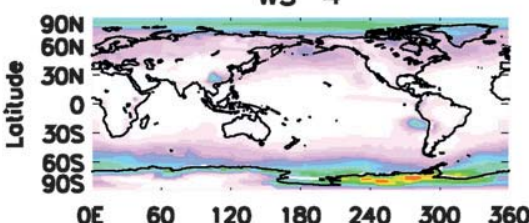

wS 7

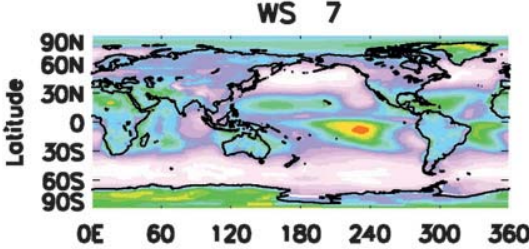

wS 10

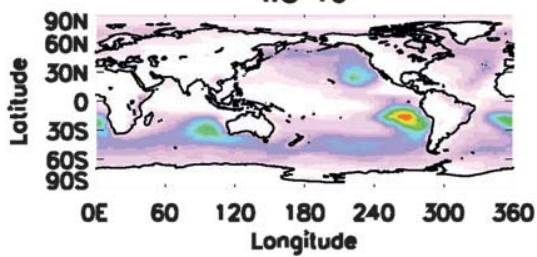

wS 2

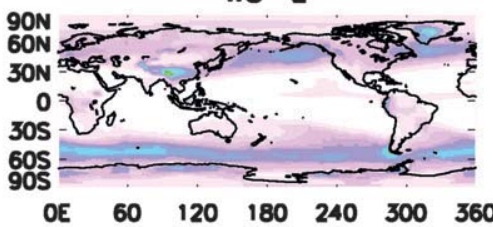

wS 5

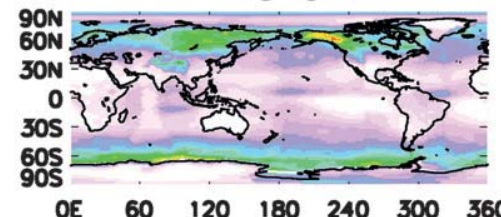

wS 8

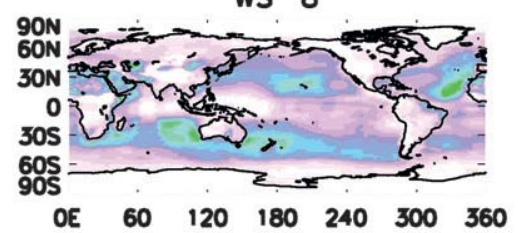

wS 11

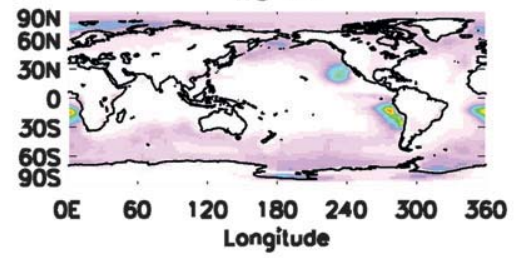

RFO (in \%)
WS 3

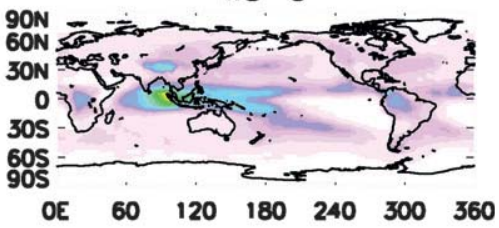

wS 6

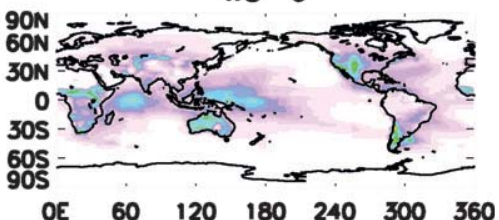

wS 9

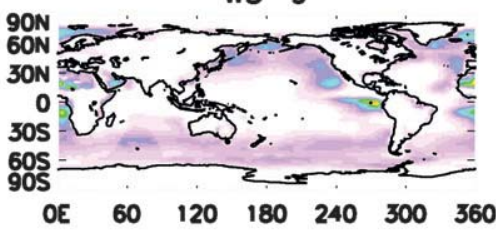

WS 12 (clear sky)

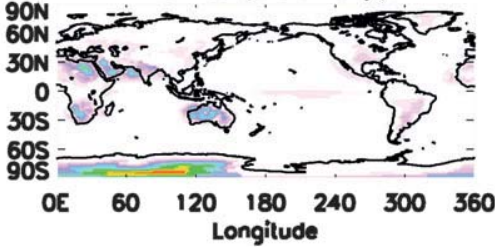

\begin{tabular}{|cccccccccc}
\hline \multicolumn{10}{|c}{ RFO (in \%) } \\
\hline
\end{tabular}

FIG. 3. Geographical distribution of the 11 WSs (clusters) as well as the totally clear sky cluster (WS 12). The colors indicate the frequency of occurrence in percent of a WS at a particular location.

Southeast Asia may reflect a "confusion" between cloud and heavy pollution events, or it may be indicative of pollution aerosol effects on cloud properties. The peak off the coast of Chile appears to be a form of marine stratus that has unusually higher cloud tops [this may be due to a low PC bias in the ISCCP product in this regime caused by biases in the atmospheric temperature profiles used to convert cloud top temperature to pressure (cf. Stubenrauch et al. 1999)]. The WS 4 RFO is $4.7 \%$ and the average CF is $92 \%$. WS 5 also includes primarily midlevel clouds, but they are optically thinner than WS 4 and are mixed with more thin cirrus clouds (Fig. 2, Table 1). These clouds are found primarily in the Southern Ocean storm track near the edge of the Antarctic ice cap, in the Siberia and Alaska regions, and in smaller concentrations in the Northern Hemisphere oceanic storm tracks and the ITCZ region. The WS $5 \mathrm{RFO}=11.5 \%$ and the average $\mathrm{CF}=83 \%$ (Table 1 ). WS 6 includes primarily high and very thin clouds classified by ISCCP as cirrus (Fig. 2, Table 1). This WS is found primarily in the Pacific

TABLE 1. Averaged PC, TAU, and total CF (\%) of mean centroid for each of the $11 \mathrm{WSs}$ based on grid-center PC and TAU and radiatively linear weighting by $\mathrm{CF}$.

\begin{tabular}{lrrrrrrrrrrr}
\hline \hline & WS 1 & WS 2 & WS 3 & WS 4 & WS 5 & WS 6 & WS 7 & WS 8 & WS 9 & WS 10 & WS 11 \\
\hline Avg PC & 275.00 & 455.00 & 355.00 & 620.00 & 550.00 & 315.00 & 600.00 & 780.00 & 825.00 & 720.00 & 735.00 \\
Avg TAU & 12.39 & 10.97 & 3.40 & 11.16 & 3.25 & 1.62 & 4.04 & 2.96 & 6.57 & 4.95 & 11.35 \\
Total CF & 98.81 & 96.83 & 93.44 & 91.56 & 83.45 & 76.42 & 29.84 & 61.85 & 81.18 & 83.09 & 93.26 \\
\hline
\end{tabular}


warm pool, Indian Ocean, and equatorial Africa regions (the lower RFO over Amazonia is because of the strong seasonality of cirrus there), where the tropical convective WS 1 and WS 3 are also present. WS 6 is also found over the major mountain ranges, primarily the Rockies, Andes, and the northern part of the Himalayas, where orographic cirrus are expected to occur more frequently. This particular cirrus pattern mixed with somewhat optically thicker high-level and midlevel clouds also occurs over the Australian and South African deserts (other desert cirrus are found in WS 7 discussed next). The WS 6 $\mathrm{RFO}=7.6 \%$ and the average $\mathrm{CF}=76 \%$ (Table 1 ). This WS was also found in the tropical analysis of Rossow et al. (2005a) with very similar cloud structure and geographical distribution.

Note that the radiation-based ISCCP retrievals of midlevel cloud top pressure result with some frequency from the combination of thin cirrus clouds overlying thicker low-level clouds. The effect that this has on the WS classification is explored further in the next section, using the WS composites of the CloudSat-CALIPSO retrievals of CVS.

The most frequently observed GWS is WS $7(\mathrm{RFO}=$ $32.5 \%$ ), which shows a peak in the PC-TAU histogram for the highest and optically thinnest clouds (Fig. 2) with a secondary peak for the lowest and slightly optically thicker clouds, classified by ISCCP as cirrus and cumulus, respectively. However, this WS also shows very infrequent occurrences of clouds at all levels and with larger TAU values, including some very thick low-level clouds. What makes this particular WS distinctive is the very low average $\mathrm{CF}$ of $30 \%$ (Table 1 ), meaning that it includes on average about $70 \%$ of clear-sky pixels in the $2.5^{\circ}$ grid cell. This WS, because of its high clear-sky fraction and predominantly optically thin clouds, will be referred to as the fair-weather WS. It occurs primarily over the tropical and subtropical oceans away from the convective regions, the Sahara and other major deserts (including those with frequent occurrences of WS 6), the ice-capped landmasses of Greenland and Antarctica, and over the Arctic Ocean with WS 4. WS 7 is also frequent over all continental regions. WS 7 has a similar geographic distribution at low latitudes as the tropical scattered cumulus WS of Rossow et al. (2005a), but that tropical WS had a more pronounced CF peak in the low and thin cloud category (cumulus) while the GWS has a larger CF peak for the highest and thinnest cloud category (cirrus). The location of the latter clouds is probably too high in the ISCCP results because their very low optical thickness does not allow a reliable retrieval of their top pressure (cf. Luo et al. 2002).

In the low-cloud GWS categories, WS 8 is dominated by low-level, optically thin clouds (shallow cumulus in the ISCCP classification), along with occasional very thin middle and high clouds. This shallow-cumulus WS has the thinnest clouds of the four low-cloud WSs and the lowest $\mathrm{CF}$ of $62 \%$ (Table 1 ). It occurs primarily over the subtropical oceans and the subtropical edges of the midlatitude storm tracks, but it is also widespread over most ocean regions and continents with an RFO of $10.4 \%$. The last three low-cloud GWSs, namely, WS 9, WS 10, and WS 11, are all dominated by low-topped clouds that have progressively larger $\mathrm{CF}$ and different average PC and TAU values (Table 1); we associate these WSs with marine stratocumulus and stratus cloud decks. This interpretation is supported by their almost exclusive occurrence over oceans, particularly off the western coastlines in the subtropics, locations that are well known for the existence of extensive stratocumulus decks. All three of these WSs also occur with lower frequencies in the midlatitude storm track regions, especially in the Southern Hemisphere. The different RFO patterns of these WSs relate primarily to the location and season of their peak occurrence. Among this group, WS 9 has the highest cloud top pressures and second largest TAU on average (Table 1) and occurs mostly in the tropics and on the equatorward side of the subtropical marine low-level cloud zones with peak frequency in the June-August (JJA) [and a secondary peak in September-November (SON)]. WS 10 is the most frequent of these low-level cloud WSs (RFO $=6.2 \%$, compared with WS $9 \mathrm{RFO}=4.4 \%$ and WS $11 \mathrm{RFO}=$ $2.6 \%$; Table 1), showing persistent, large concentrations off the western subtropical coasts of North and South America, Africa, and Australia. The seasonal variation of WS 10 RFO (not shown) peaks in JJA off the California coast but exhibits little seasonal variation everywhere else. Significant amounts of WS 10 also occur on the subtropical edges of the midlatitude storm tracks. Finally, WS 11 includes the optically thickest low cloud with the smallest amount of clear sky (Table 1) and occurs mostly in JJA and SON located very close to the continental coasts. All three stratocumulus WSs have average $\mathrm{CF}>80 \%$ (WS 9 is $81.2 \%$, WS 10 is $83 \%$, and WS 11 is $93 \%$; Table 1 ).

The clear-sky WS 12 represents environments where the $2.5^{\circ}$ ISCCP map grid cell is completely cloud-free. This WS occurs primarily over the Antarctic and the Sahara, Kalahari, Arabian, and Australian deserts. The clear-sky WS 12 RFO is only about $2 \%$. The clear-sky grid cells were predefined as a separate WS and were not included in the cluster analysis, as the objective was to cluster cloud structures. A test of their inclusion in the analysis produced very similar cluster distributions and included most of the clear scenes in WS 7 and, secondarily, WS 6 . Note that the 12 GWSs comprise $100 \%$ of 

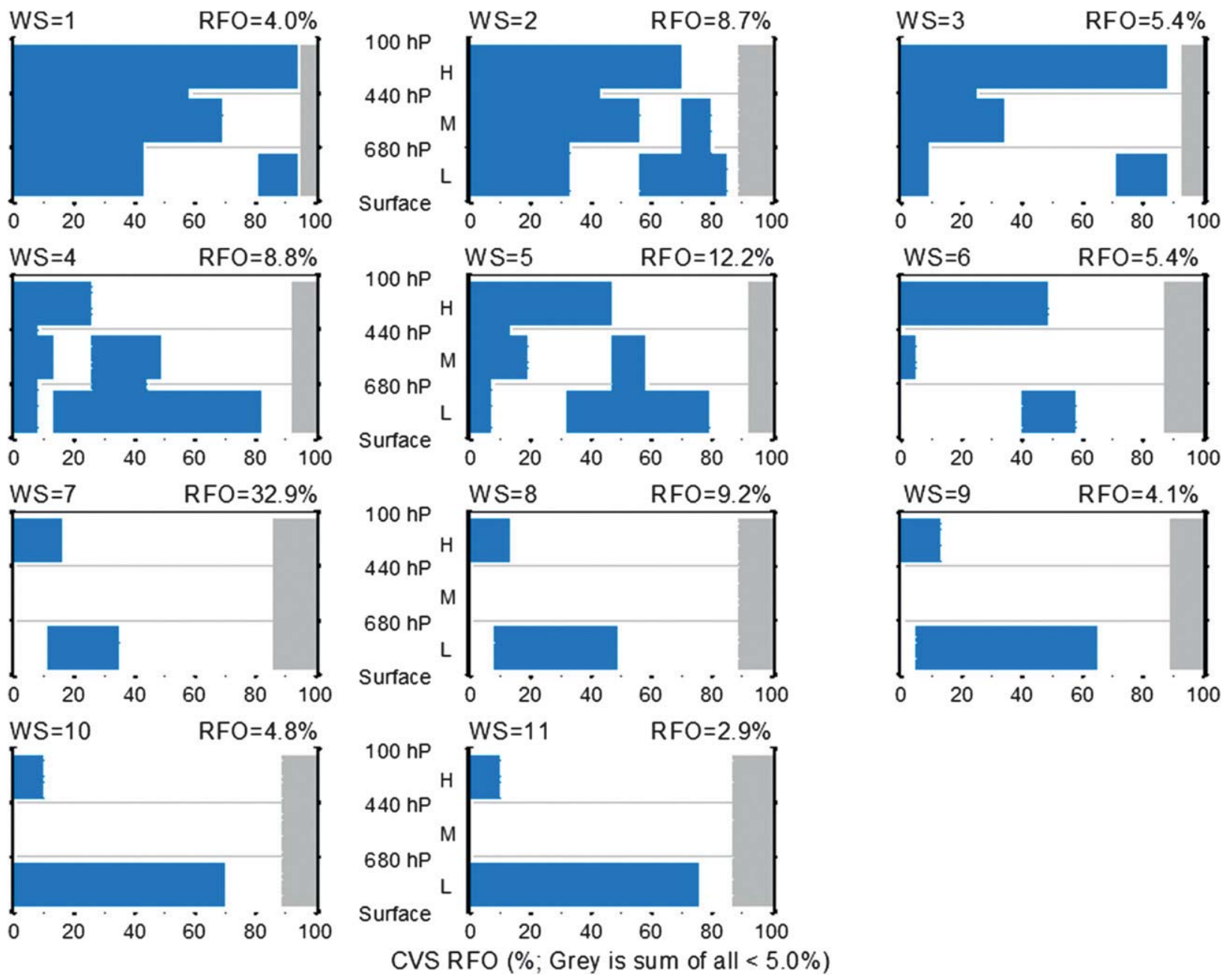

CVS RFO (\%; Grey is sum of all $<5.0 \%$ )

FIG. 4. CVS distributions for the 11 WSs. The width of each CVS bar indicates the frequency of occurrence of this CVS in the particular WS. The white bar (space) indicates clear sky, and the gray bar represents the sum of all CVSs that occur less than $5 \%$ of the time.

the global domain over the 26 years of the ISCCP dataset, excluding only the time periods with no sunlight when optical depth retrievals are not made by the ISCCP analysis.

\section{b. CloudSat-CALIPSO cloud vertical structure of the weather states}

As detailed in the previous section, $C-C$ cloud vertical profiles are classified into one of 11 CVS types (see Fig. 1, including clear sky) and matched in time and space to the GWS. As there can be multiple $C-C$ profiles in a single $2.5^{\circ}$ map grid cell, we include all profiles to produce a statistical distribution of CVS for each GWS. Figure 4 shows the RFO of each CVS type composited for each GWS: the horizontal axis is the fraction of the $C-C$ profiles in each CVS type that co-occur with each GWS, where only the CVS types that occur in $\geq 5 \%$ of the matchups are shown. The sum of all the types that occur less that $5 \%$ of the time is shown by the gray bar at the right end of the graph. Clear-sky fraction is indicated by the white bar. Note that these results come from the 3.5-yr period, July 2006-December 2009, when both ISCCP and $C-C$ are available. This explains why the GWS RFO values shown in Fig. 4 are not the same as those shown in Fig. 2.

The interpretation of WS 1 and WS 2 as being dominated by deep convection is confirmed by the $C-C$ profiles in Fig. 4, where $30 \%-40 \%$ of the profiles co-occurring with these WSs are classified as a cloud extending from the lower atmosphere continuously to the upper troposphere (HxMxL). The tropical WS 1 has about 10\% more deep convective cloud (HxMxL, see Fig. 1) than WS 2 and also has somewhat more clouds that extend from midlevels into the upper troposphere (thick stratiform anvil, HxM, see Fig. 1). WS 1 has somewhat less high-middle two-layer clouds (HM) but more isolated 
high clouds $(1 \mathrm{H})$ than WS 2. Both WS 1 and WS 2 have about $10 \%$ of high cloud overlying low clouds (HL), which in the ISCCP retrieval can appear as middle clouds, but WS 2 actually includes about $10 \%$ of actual middle clouds that extend from the lower levels into the midlevels (MxL, Fig. 1), which WS 1 lacks. This excess thick middle cloud in WS 2 is also apparent in the WS 2 PC-TAU histogram (Fig. 2). WS 2 also includes a larger sum $(\sim 15 \%)$ of CVS types that each occurs less than $5 \%$ of the time than WS 1 . All of these differences in the $C-C$ CVS type distribution reinforce the original PC-TAUbased distinction of these two WSs. The $C-C$ profiles also confirm that WS 3 contains much less deep convection (only 10\% of HxMxL), a similar amount of thick stratiform anvil cloud (nearly $20 \%$ of $\mathrm{HxM}$ ), and a lot more isolated high-level cloud (about $35 \%$ of $1 \mathrm{H}$ ) than either WS 1 or WS 2. All these CVS types would fall into the cirrus and anvil categories that dominate the PC-TAU histograms of WS 3 (Fig. 2). The $10 \%$ of deep convection in WS 3 is consistent with the interpretation that these are isolated convective plumes (cf. Rossow et al. 2005a).

The polar WS 4 appears in the $C-C$ profiles as a more complicated mixture of many CVS types, including more of the low to midlevel (MxL) than any other weather state ( $20 \%)$, some "deep convection" (about $7 \% \mathrm{HxMxL}$, but note that the tropopause height is much lower in the polar regions so that this cloud type appears as very optically thick clouds with midlevel PC in Fig. 2), and a little more than $30 \%$ of isolated low-level cloudiness (1L). WS 4 also contains more varieties of multilayer CVS types than any other WS, including 7\% HM, $15 \% \mathrm{HL}$, and $7 \% \mathrm{ML}$. The key conclusion is that the $C-C$ profiles confirm the preponderance of midlevel cloud tops for this polar WS.

WS 5, which occurs primarily in the poleward part of the midlatitude storm tracks, contains about $20 \%$ of HL cloud and $10 \%$ of $\mathrm{MxL}$ cloud that together explain the predominance of midlevel cloud in the PC-TAU histogram (Fig. 2). In addition, it contains $\sim 20 \%$ of isolated low-level (1L) and isolated cirrus $(1 \mathrm{H})$ cloud and less that $10 \%$ of cloud extending from low to high in the troposphere. The "cirrus" WS 6, associated mostly with tropical convection, is dominated in the ISCCP classification by optically thin, high-level clouds with some middle- and lower-level clouds; the $C-C$ profiles confirm that WS 6 is associated almost entirely with isolated high-level clouds (CF $235 \%$ of $1 \mathrm{H}$ ) with about $10 \%$ of $\mathrm{HL}$, as well as about $30 \%$ clear sky, close to the climatological value in Table 1 $(\sim 24 \%)$. This WS also has a large sum $(\sim 13 \%)$ of a variety of CVS types that each occur $<5 \%$ of the time.

The fair-weather WS 7 is confirmed by $C-C$ to be largely clear sky (54\% as compared to about $70 \%$ in Table 1 ) with the dominant CVS types being isolated high $(1 \mathrm{H})$ and isolated low (1L) clouds; there is also about 7\% of HL and about $15 \%$ of a variety of rare CVS types. This is the same mixture of isolated cirrus and cumulus exhibited by the PC-TAU histogram (Fig. 2). All of these features suggest a weak dynamic regime with small winds and only a "debris" collection of different cloud types.

The $C-C$-based CVS types associated with WSs 8-11 are all dominated by isolated low-level clouds (1L) accompanied by some (about $10 \%-15 \%$ ) $1 \mathrm{H}$ or HL profiles. A notable feature is that the fraction of $1 \mathrm{~L}$ cloudiness increases and the fraction of cirrus and clear sky decreases going from WSs 8-11. All of these features agree very well with the inferences based solely on the PC-TAU classification (Fig. 2 and Table 1), where the order of the WS was selected before we saw the $C-C$ results for CVS. All of the low-cloud WSs include about $10 \%$ of a mixture of rare CVS types.

In summary, the matched $C-C$-based CVS types confirm that the ISCCP GWSs are indeed distinct situations with different cloud vertical structures as well as different horizontal structures. The composites of CVS also help clarify which of the midlevel clouds derived from passive radiation measurements result from high, thin clouds overlying low clouds and which are actual middle-topped clouds. The combination of the PC-TAU WS categories and the matched CVS type distributions provide a clearer picture of the 3D cloud structure of the major atmospheric weather states.

\section{Discussion}

The cluster analysis applied to the ISCCP PC-TAU histograms produced a comprehensive classification of the global atmosphere into 12 GWSs as expressed by distinctive mesoscale distributions of cloud properties. Matching those WSs with cloud vertical profiles from CloudSat and CALIPSO that are classified into $11 \mathrm{CVS}$ types showed that each of the ISCCP GWSs is associated with distinctive distributions of the CVS types. A number of the subtle differences among the GWSs were confirmed and further clarified by these CVS distributions. For example, the global cluster analysis distinguished between tropical deep convection and similar high-topped, optically thick clouds that occur in midlatitude storms but indicated differences in cloud top height and in the proportions of cirrus and midlevel clouds that extend to low levels. The global cluster analysis also identified a unique high-latitude WS and a fair-weather WS, which the CVS profiles show to be a mixture of "debris" clouds with shallow cumulus and large clear-sky fractions. Finally, the several WSs dominated by low-level clouds were shown to have systematically different amounts of low-level clouds and clear sky by $C-C$. 


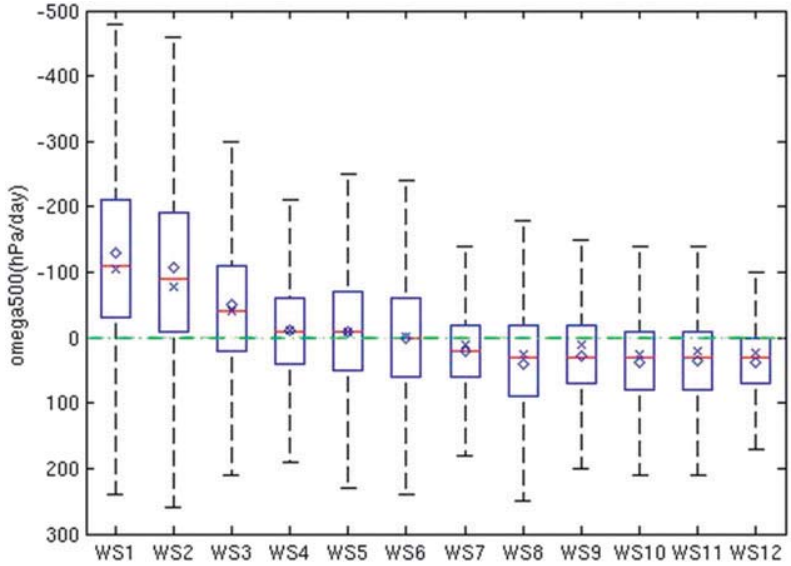

FIG. 5. Box-and-whisker diagram of ERA-Interim 500-mb vertical velocity distributions for the 11 WSs. The line represents the median, the rhombus represents the mean, the box represents the 75th percentile, and the bar represents the extremes of each distribution. The " $\times$ " symbol represents the mean from an analysis of NRA2 500-mb vertical velocity.

Two regional studies have shown associations between the cloud-based WSs and the dynamic and thermodynamic conditions (Jakob et al. 2005; Haynes et al. 2011). We expand on this analysis approach by compositing the WS-associated 500 -mb vertical velocities $\left(W_{500}\right)$ from two reanalyses to represent the dynamic conditions producing each WS and CVS distribution. We match the GWSs at 6-h intervals with $W_{500}$ values from the ERA-Interim reanalysis. Figure 5 shows a boxand-whisker representation of the $W_{500}$ distributions for each of the GWSs, where the line is the median value and the open diamond shape is the mean value. Also shown by an " $X$ " symbol is the mean value from the NRA2 reanalysis. A very regular progression can be observed, with the more convective WSs showing the largest upward median and mean values of $W_{500}$ and the low-cloud categories showing small downward mean and median $W_{500}$ values. Tropical deep convection (WS 1), midlatitude storm clouds (WS 2), and anvil clouds and isolated convection (WS 3) show upward mean $W_{500}$ between -50 and $-130 \mathrm{hPaday}^{-1}$. The polar and high-latitude WS categories (WS 4 and WS 5) have mean and median values around $-10 \mathrm{hPa}^{-1}$ and $^{-1}$ and distributions more weighted toward the upward motions. The cirrus WS 6 has mean and median $W_{500}$ right at zero and a distribution equally weighted between upward and downward motions. The fair-weather WS 7 has mean $W_{500}$ values around $+20 \mathrm{hPa} \mathrm{day}^{-1}$ and a distribution skewed toward downward motions. The low-cloud WSs all have downward mean $W_{500}$ between +25 and $30 \mathrm{hPa} \mathrm{day}^{-1}$, as does the clear-sky WS 12 . The fact that the mean and median values are close in most of the distributions implies normally weighted distribution shapes. The results from the NRA2 reanalysis are very similar to those from the ERA-Interim reanalysis, but the quantitative difference suggests that the NRA2 circulation is generally weaker than the ERA-Interim circulation.

The WSs in this study are derived from PC-TAU histograms in the ISCCP D1 dataset but are different from the cloud types defined there by the specific combinations of PC and TAU values (Rossow and Schiffer 1999). Instead, the WSs can be thought of as representing distinctive mesoscale distributions or mixtures of these cloud types. The important difference is that the occurrence of WSs does not depend as much on the absolute values of PC and TAU, only on the resemblance of the histogram patterns, whereas the amounts of each cloud type depend on specific PC and TAU ranges. The only preimposed condition on the cluster statistics is the total number of clusters that is derived using objective criteria (see section 2). Hence, the results for the RFO of the WSs are not sensitive to small calibration changes and have little dependence on satellite view angle. This makes the WSs a better tool than cloud amount or cloud type to examine cloud trends and long-term variability. In a previous study (Tselioudis et al. 2010), the anomaly time record of the tropical WS 1 was used to explain the variability of lower stratospheric water vapor found in satellite observations and used to explain the 1990s fast warming rate (Solomon et al. 2010). In this study, the anomaly time records of the 11 GWSs over 26 years (1983-2009) are shown in Fig. 6. The deep convection WS 1 shows a small decrease of about $0.3 \%$ in the first part of the period and a more notable increase of about $0.5 \%$ between 1995 and 2005. This behavior is similar to that described in Tselioudis et al. (2010), where only the tropical component $\left(15^{\circ} \mathrm{S}-15^{\circ} \mathrm{N}\right)$ of the deep convective WS was analyzed. The midlatitude storm cloud WS 2 shows a notable increase of about 2\% between 1995 and 2010, which comes mostly from increases of this WS in the poleward part of the storm tracks (result not shown). This is in agreement with the results of Bender et al. (2012) that showed a poleward shift in ISCCP total cloudiness in the same time period. The polar WS 4 also shows an increase of about $2 \%$ between 1995 and 2010, while the midlevel WS 5 shows a decrease of similar magnitude in the same time period.

The largest changes in the WS RFOs occur in the low cloud categories. The most-frequently occurring fairweather WS 7 shows a large increase of about $5 \%$ between 1990 and 2000, followed by a small decrease and a flattening of the curve in the last decade. At the same time, the shallow cumulus WS 8 decreased by about $4 \%$ while all three of the stratocumulus WSs show weaker decreases in the same time period. 

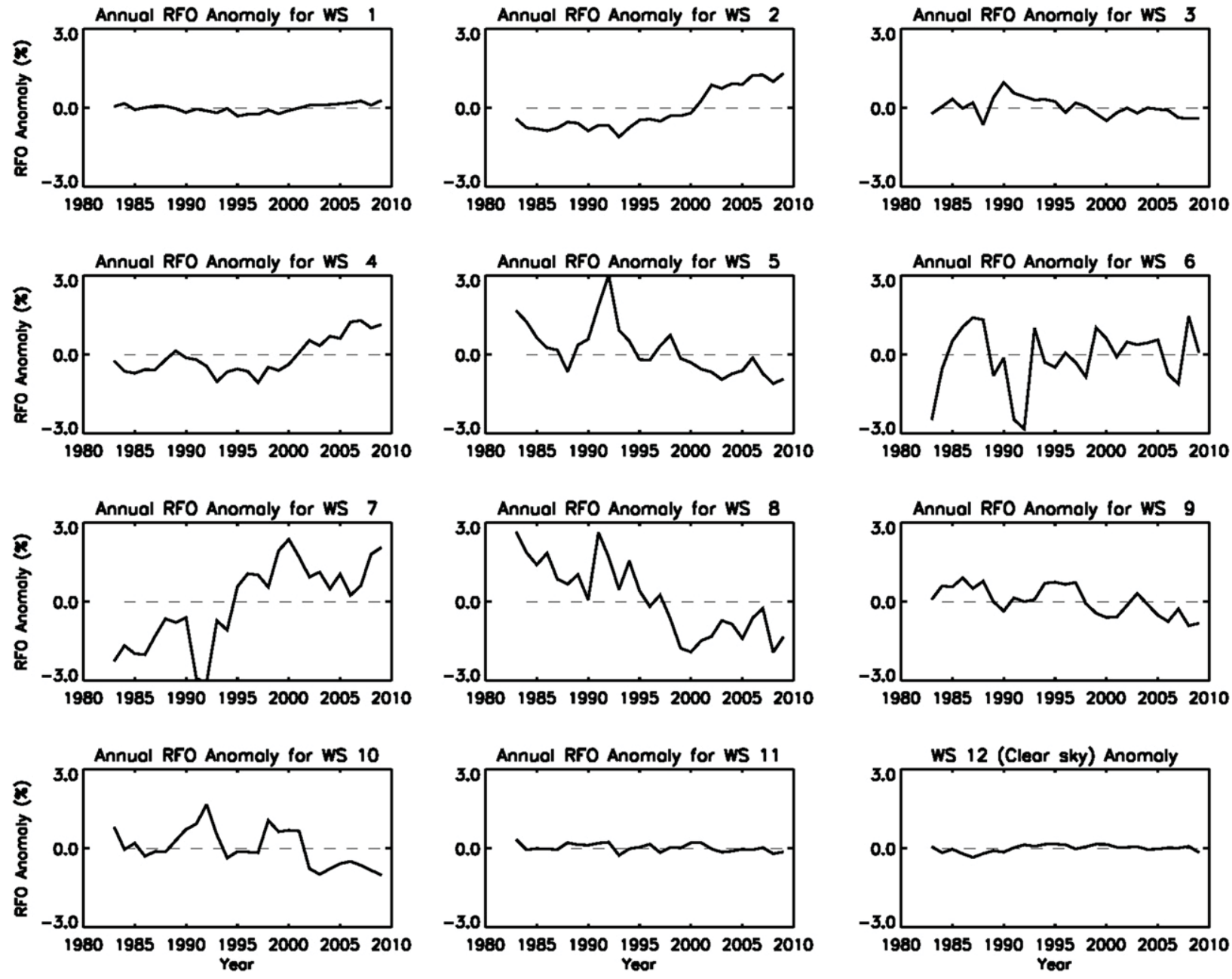

FIG. 6. Time series of annual RFO anomalies for the 11 WSs, for the period 1983-2009.

This large increase in the fair-weather WS 7 at the expense of the shallow cumulus WS 8 (and other lowlevel cloud WSs) is important from the cloud radiative feedback perspective, as this WS produces the weakest cloud radiative cooling of all WSs, a radiative cooling that is about $60 \mathrm{~W} \mathrm{~m}^{-2}$ weaker than the shallow cumulus WS 8 and $150-200 \mathrm{~W} \mathrm{~m}^{-2}$ weaker than the other lowlevel cloud WSs. This is shown in Fig. 7, which shows box-and-whisker diagrams of the top-of-the-atmosphere shortwave CRE (Fig. 7a) and the longwave CRE (Fig. 7b) of the 11 WSs. The shortwave CRE values show strong radiative cooling for the six high- and middle-cloud WSs, ranging from $350 \mathrm{~W} \mathrm{~m}^{-2}$ for the tropical convection WS to $60 \mathrm{~W} \mathrm{~m}^{-2}$ for the cirrus WS. The fair-weather WS 7 shows very weak cloud radiative cooling of only about $10 \mathrm{~W} \mathrm{~m}^{-2}$, due primarily to the large amount of clear sky that is included in it. The shallow cumulus WS 8 shows radiative cooling of about $70 \mathrm{~W} \mathrm{~m}^{-2}$, while the three stratocumulus WS show cooling between 150 and $210 \mathrm{~W} \mathrm{~m}^{-2}$.
The longwave CRE of the six high- and middle-cloud WSs shows radiative warming ranging from 20 to about $100 \mathrm{~W} \mathrm{~m}^{-2}$. The five low-cloud WSs, including the fairweather one, show weak cloud radiative warming that ranges from about $10 \mathrm{~W} \mathrm{~m}^{-2}$ to about $20 \mathrm{~W} \mathrm{~m}^{-2}$ for the last two WSs that show the highest cloud tops in their PC-TAU distributions (Fig. 2).

The WS CRE distributions indicate that the sharp increase of WS 7 in the 1990s and the flattening of that increase in the 2000s together with the opposite behavior of WSs 8-11 could have provided an additional radiative warming for the global temperature fluctuations of that period (e.g., Solomon et al. 2010; Tselioudis et al. 2010). Note that tropical shortwave radiative warming in the 1990s has also been found in several studies that analyzed Earth Radiation Budget Experiment (ERBE) and Clouds and the Earth's Radiant Energy System (CERES) radiative flux retrievals (e.g., Wielicki et al. 2002; Zhang et al. 2004; Wong et al. 2006). 

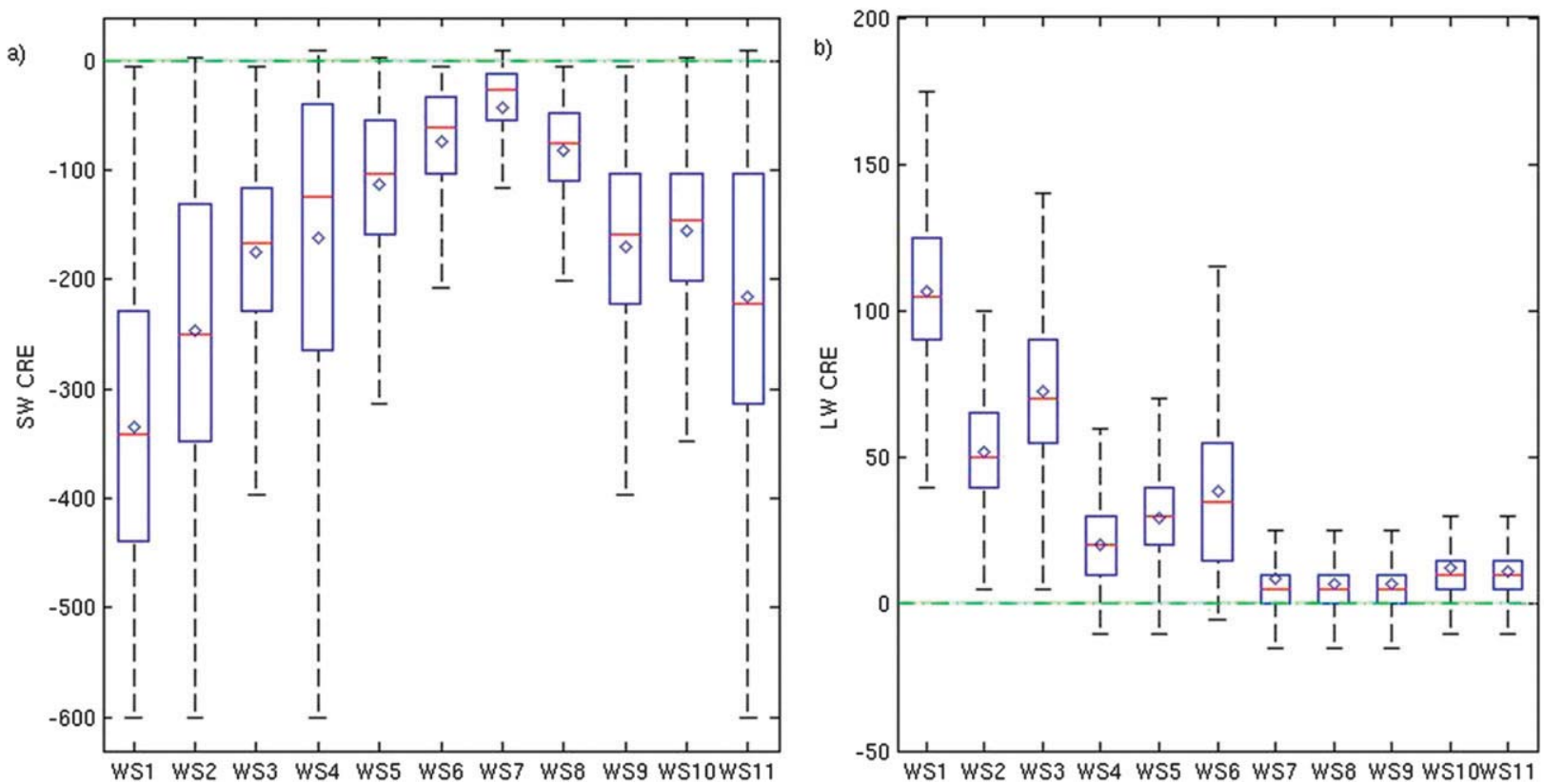

FIG. 7. Box-and-whisker diagram of ISCCP FD (a) shortwave and (b) longwave CRE distributions for the 11 WSs. The symbols are as in Fig. 5.

The cloud-based global weather states derived in this study constitute a comprehensive way to separate distinct atmospheric weather regimes and examine their properties and variability. To the extent that climate change can be viewed as a change in the relative frequency of atmospheric weather regimes, the GWSs can be used to understand the feedbacks produced by changes in the cloud structure and properties with climate change and to quantify their effect on the Earth's radiative balance. This study describes the properties and structure of the GWSs, the distribution of the CVS, their composite association with atmospheric vertical motions, and their radiative signatures, providing a preliminary examination of how the atmospheric circulation regimes connect to cloud properties and radiative heating. In future work, more complex definitions of dynamic and thermodynamic regime for the WSs will be derived, and the mechanisms producing shifts in the WS regime time and space variability will be investigated. To promote usage of the WS regimes in climate analysis studies, the WS dataset is available online (at http://isccp.giss.nasa.gov/ climanal5.html and at http://crest.ccny.cuny.edu/rscg/ products.html).

Acknowledgments. The authors of this work would like to acknowledge support by the NASA MAP and MEaSUREs programs, the NOAA CDR program, and the EU FP7 EUCLIPSE program.

\section{REFERENCES}

Anderberg, N. R., 1973: Cluster Analysis for Applications. Elsevier, $359 \mathrm{pp}$.

Bender, F. A.-M., V. Ramanathan, and G. Tselioudis, 2012: Changes in extratropical storm track cloudiness 1983-2008: Observational support for a poleward shift. Climate Dyn., 38, 2037-2053, doi:10.1007/s00382-011-1065-6.

Dee, D. P., and Coauthors, 2011: The ERA-Interim reanalysis: Configuration and performance of the data assimilation system. Quart. J. Roy. Meteor. Soc., 137, 553-597.

Haynes, J. M., C. Jakob, W. B. Rossow, G. Tselioudis, and J. Brown, 2011: Major characteristics of Southern Ocean cloud regimes and their effects on the energy budget. J. Climate, 24, 5061-5080.

Jakob, C., and G. Tselioudis, 2003: Objective identification of cloud regimes in the tropical western Pacific. Geophys. Res. Lett., 30, 2082, doi:10.1029/2003GL018367.

- - — and T. Hume, 2005: The radiative, cloud and thermodynamic properties of the major tropical western Pacific cloud regimes. J. Climate, 18, 1203-1215.

Kanamitsu, M., W. Ebisuzaki, J. Woollen, S. K. Yang, J. J. Hnilo, M. Fiorino, and G. L. Potter, 2002: NCEP-DOE AMIP-II Reanalysis (R-2). Bull. Amer. Meteor. Soc., 83, 1631-1644.

Lee, D., L. Oreopoulos, G. J. Huffman, W. B. Rossow, and I.-S. Kang, 2013: The precipitation characteristics of ISCCP tropical weather states. J. Climate, 26, 772-788.

Luo, Z., W. B. Rossow, T. Inoue, and C. J. Stubenrauch, 2002: Did the eruption of the Mt. Pinatubo volcano affect cirrus properties? J. Climate, 15, 2806-2820.

Mace, G. G., Q. Zhang, M. Vaughan, R. Marchand, G. L. Stephens, C. Trepte, and D. Winker, 2009: A description of hydrometeor layer occurrence statistics derived from the first year of merged CloudSat and CALIPSO data. J. Geophys. Res., 114, D00A26, doi:10.1029/2007JD009755. 
Mekonnen, A., and W. B. Rossow, 2011: The interaction between deep convection and easterly waves tropical North Africa: A weather state perspective. J. Climate, 24, 4276-4294.

Oreopoulos, L., and W. B. Rossow, 2011: The cloud radiative effect of International Satellite Cloud Climatology Project weather states. J. Geophys. Res., 116, D12202, doi:10.1029/ 2010JD015472.

Rossow, W. B., and R. A. Schiffer, 1999: Advances in understanding clouds from ISCCP. Bull. Amer. Meteor. Soc., 80, 2261-2287.

_ - and Y. Zhang, 2010: Evaluation of a statistical model of cloud vertical structure using combined CloudSat and CALIPSO cloud layer profiles. J. Climate, 23, 6641-6653.

— G. Tselioudis, A. Polak, and C. Jacob, 2005a: Tropical climate described as a distribution of weather states indicated by distinct mesoscale cloud property mixtures. Geophys. Res. Lett., 32, L21812, doi:10.1029/2005GL024584.

_ - Y.-C. Zhang, and J.-H. Wang, 2005b: A statistical model of cloud vertical structure based on reconciling cloud layer amounts inferred from satellites and radiosonde humidity profiles. J. Climate, 18, 3587-3605.

—, A. Mekonnen, C. Pearl, and W. Goncalves, 2013: Tropical precipitation extremes. J. Climate, 26, 1457-1466.

Solomon, S., K. Rosenlof, R. Portmann, J. Daniel, S. Davis, T. Sanford, and G.-K. Plattner, 2010: Contributions of stratospheric water vapor to decadal changes in the rate of global warming. Science, 327, 1219-1223, doi:10.1126/ science. 1182488.

Stubenrauch, C. J., W. B. Rossow, F. Cheruy, A. Chédin, and N. A. Scott, 1999: Clouds as seen by satellite sounders (3I) and imagers (ISCCP). Part I: Evaluation of cloud parameters. J. Climate, 12, 2189-2213.

Tan, J., and C. Jakob, 2013: A three-hourly dataset of the state of tropical convection based on cloud regimes. Geophys. Res. Lett., 40, 1415-1419, doi:10.1002/grl.50294.

_ _ - and T. P. Lane, 2013: On the identification of the largescale properties of tropical convection using cloud regimes. J. Climate, 26, 6618-6632.
Tromeur, E., and W. B. Rossow, 2010: Interaction of tropical deep convection with the large-scale circulation in the MaddenJulian oscillation. J. Climate, 23, 1837-1853.

Tselioudis, G., and W. B. Rossow, 2011: Time scales of variability of the tropical atmosphere derived from clouddefined weather states. J. Climate, 24, 602-608.

_ E. Tromeur, W. B. Rossow, and C. S. Zerefos, 2010: Decadal changes in tropical convection and their effects on stratospheric water vapor. Geophys. Res. Lett., 37, L14806, doi:10.1029/ 2010GL044092.

Wang, J., W. B. Rossow, and Y.-C. Zhang, 2000: Cloud vertical structure and its variations from a 20-year global rawinsonde dataset. J. Climate, 13, 3041-3056.

Wielicki, B. A., and Coauthors, 2002: Evidence for large decadal variability in the tropical mean radiative energy budget. Science, 295, 841-844.

Williams, K. D., and G. Tselioudis, 2007: GCM intercomparison of global cloud regimes: Present-day evaluation and climate change response. Climate Dyn., 29, 231-250, doi:10.1007/ s00382-007-0232-2.

— , and M. J. Webb, 2009: A quantitative performance assessment of cloud regimes in climate models. Climate Dyn., 33, 141-157, doi:10.1007/s00382-008-0443-1.

Wong, T., B. A. Wielicki, R. B. Lee III, G. L. Smith, K. A. Bush, and J. K. Willis, 2006: Reexamination of the observed decadal variability of the earth radiation budget using altitudecorrected ERBE/ERBS nonscanner WFOV data. J. Climate, 19, 4028-4040.

Zhang, Y., W. B. Rossow, A. A. Lacis, V. Oinas, and M. I. Mishchenko, 2004: Calculation of radiative fluxes from the surface to top of atmosphere based on ISCCP and other global data sets: Refinements of the radiative transfer model and the input data. J. Geophys. Res., 109, D19105, doi:10.1029/ 2003JD004457.

_ , S. A. Klein, G. G. Mace, and J. Boyle, 2007: Cluster analysis of tropical clouds using CloudSat data. Geophys. Res. Lett., 34, L12813, doi:10.1029/2007GL029336. 